\title{
The vascular patterns of the posterior part of the circulus arteriosus cerebri (Willisi)
}

\author{
El Falougy H, Weismann P, Lukacikova P, Mifkovic A, Perzelova A, Sivakova I, Kubikova E \\ Institute of Anatomy, Faculty of Medicine, Comenius University in Bratislava. \\ eliska.kubikova@fmed.uniba.sk
}

\begin{abstract}
BACKGROUND: Various authors defined three patterns of the posterior part of the circulus arteriosus cerebri Willisi $(\mathrm{CW})$ according to the diameter of the posterior communicating artery (PCoA) and the precommunicating segment of the posterior cerebral artery (P1). In the adult pattern, the P1 has a diameter larger than the nonhypoplastic PCOA. In the transitional pattern, the diameter of the PCOA is equal to that of the P1. In the fetal pattern, the diameter of the P1 is smaller than the diameter of the PCoA. The study was aimed to evaluate the configurations and calibers of the posterior part of the CW.

METHODS: The work was conducted on 185 adult post-mortem brains. The CW and its branches were photographed by a digital camera. We used the software Image $\mathrm{J}$ to evaluate and process the gained images.

RESULTS: The fetal pattern was found unilaterally in $8.37 \%$, and bilaterally in $4.86 \%$. The transitional pattern was observed unilaterally in $6.47 \%$, and bilaterally in $1 \%$. The prevalence of the unilateral and bilateral adult patterns was equal (21.62 \% for each configuration). The hypoplastic PCoA was found unilaterally in $17.57 \%$, and bilaterally in $16.76 \%$. CONCLUSION: Various factors including genetic and environmental may affect the development of the cerebral vessels and their dimensions. The distinguishing of the vascular dimensions in vivo can help in the expectation and may be the avoidance of possible cerebrovascular disturbances in the future. Correlation and interdisciplinary cooperation of the studies dealing with morphology, radiology, and hemodynamics of the cerebral vessels are becoming an urgent need. The assumed results of this cooperation can be used in tabulating the calibers of the cerebral vessels and determining the threshold dimensions under which failure of hemodynamics and collateral function may appear (Tab. 2, Fig. 5, Ref. 28). Text in PDF www.elis.sk.

KEY WORDS: circulus arteriosus cerebri, vascular patterns, morphometry, hypoplasia, fenestration.
\end{abstract}

\section{Introduction}

The posterior cerebral artery (PCA) is the terminal branch of the basilar artery (BA) at the level of the interpeduncular fossa. It is divided according to its attachment to the posterior communicating artery (PCoA) into the pre-communicating part (P1) and the post-communicating part (P2) (1).

The paired PCoA is the collateral vessel between the internal carotid and vertebrobasilar systems. In the physiological cases, the pressure of streams from both systems will be equal in the PCoA causing almost no mixture of blood. On the other hand, if any of the systems does have an obstructive disease; blood will flow from the normal system to the one with lower pressure through the communicating arteries $(2,3,4)$

The most commonly appearing variation of the arterial caliber between individuals occurs in the PCoA (1). Various authors dis-

Institute of Anatomy, Faculty of Medicine, Comenius University in Bratislava, Sasinkova 2, 81372 Bratislava, Slovakia

Address of correspondence: E. Kubikova, MD, PhD, Institute of Anatomy, Faculty of Medicine, Comenius University in Bratislava, Sasinkova 2, SK-813 72 Bratislava, Slovakia. Phone: +421.2.59357390

Acknowledgement: This work was supported by the Project 1/0959/16 of the Grant Agency of the Ministry of Education and Slovak Academy of Sciences (VEGA). tinct and describe three patterns of the posterior part of the circlus arteriosus cerebri Willisi (CW) on the base of the vascular caliber of the PCoA and the P1 segment. In the adult pattern, the P1 has a diameter larger than the non-hypoplastic PCoA. In the transitional pattern, the diameter of the PCoA is equal to that of the P1. In the fetal pattern, the diameter of the $\mathrm{P} 1$ is smaller than the diameter of the PCoA (5, 6, 7) (Fig. 1).

The diverse patterns of the posterior part of the CW might have a different effect on the cerebral circulation during the pathological processes. The present work focuses on studying and evaluating the variable vascular configurations of the posterior part of the $\mathrm{CW}$ and their various morphometric characteristics.

\section{Materials and methods}

The study was conducted on 185 adult post-mortem brains (370 hemispheres), which were obtained during the anatomical autopsies at the Faculty of Medicine, Comenius University in Bratislava. The brains were removed from the cranial cavity and preserved in a solution of formalin (38\% formaldehyde) and alcohol (isopropyl alcohol). The arachnoid was thoroughly dissected from each cerebral base to evaluate the vessels of the brain. The CW and its branches were photographed with an Olympus digital camera (Camedia C-5050). We used the image processing software Image 

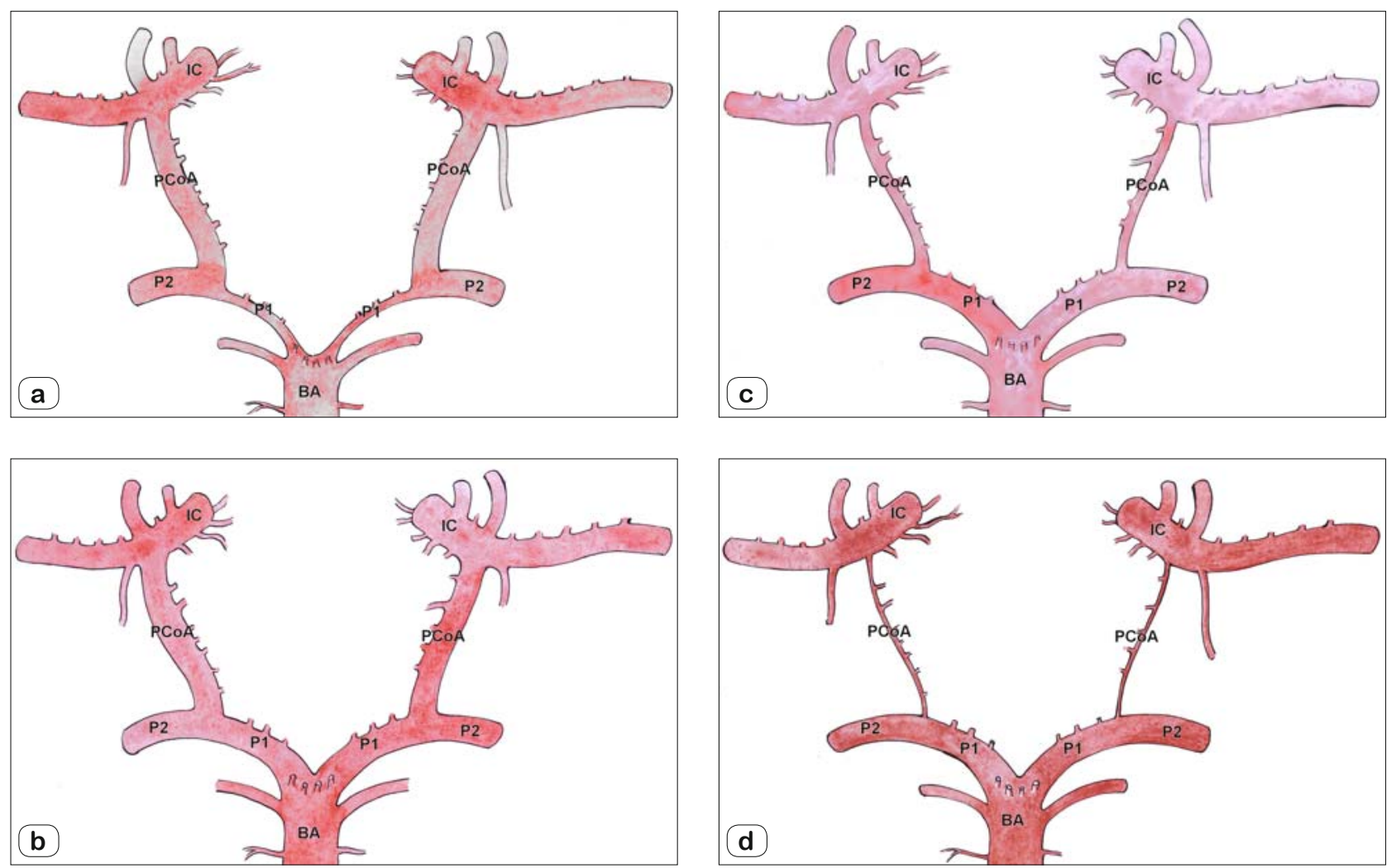

Fig. 1. The vascular patterns of the posterior part of the circulus arteriosus cerebri. (a) Bilateral fetal pattern. (b) Bilateral transitional pattern. (c) Bilateral adult pattern. (d) Bilateral hypoplasia of the posterior communicating artery (PCoA). ICA - internal carotid artery; P1 - precommunicating segment of the posterior cerebral artery; P2 - postcommunicating segment of the posterior cerebral artery; BA - basilar artery.

J (U.S. National Institutes of Health) to evaluate the external vascular values (length and the external diameter). Vascular calibers were measured according to the metric scale, which was applied in situ. The scale established the known metric measure in each image, according to which the software was able to determine the metric distances of the image by computing the pixel differences.

\section{Results}

Several morphological studies defined the hypoplastic PCoA as a vessel with a diameter lesser than $1 \mathrm{~mm}$. The authors of various radiological studies considered the lesser threshold of the hypoplastic vessels ranged between $0.4-0.8 \mathrm{~mm}(2,8,9)$. The brains used in this study were fixed in formalin and alcohol; therefore, we calculated with a reduction in the vascular calibers (10). Due to this fact, we determined the $0.8 \mathrm{~mm}$, limit as the threshold, under which the artery was indicated as hypoplastic.

The mean outer diameter of the right PCoA was $1.1 \mathrm{~mm}$ with a range from 0.34 to $3.37 \mathrm{~mm}$. The mean diameter of the left PCoA was $1.04 \mathrm{~mm}$ with a range $0.35-2.78 \mathrm{~mm}$. The hypoplasia of the PCoA was seen bilaterally in 31 brains (16.76\%). The unilateral hypoplastic artery was found in $17.57 \%$ of cases. The left-sided hypoplasia was distinguished in 36 hemispheres (9.73\%). The right hypoplastic PCoA appeared in 29 hemispheres (7.84 \%). The PCoA was absent in $1.62 \%$ of cases. The aplasia of the artery occurred

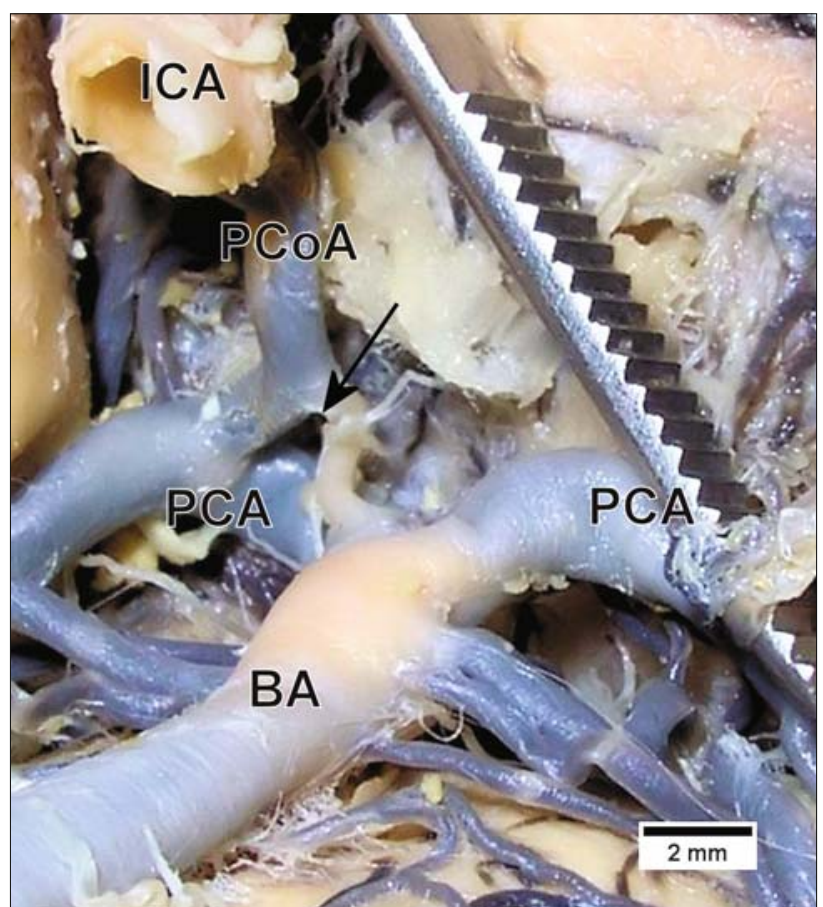

Fig. 2. Fenestration of the right posterior communicating artery $(\mathrm{PCoA})$ is signed by a black arrow. ICA - internal carotid artery; PCA - posterior cerebral artery; BA - basilar artery. 


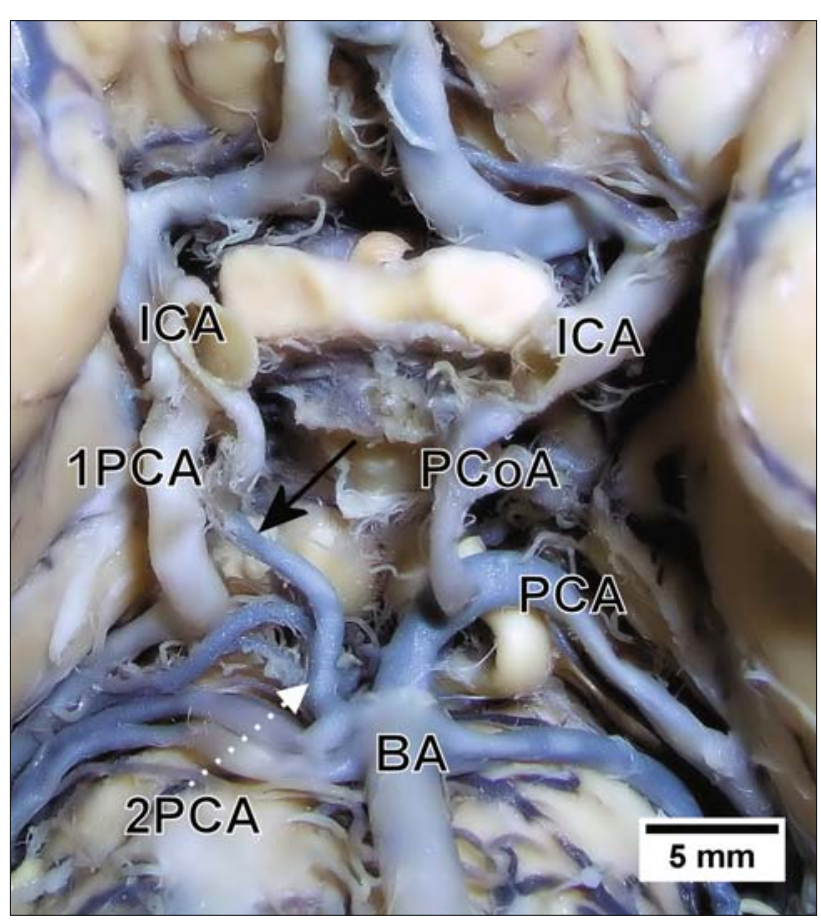

Fig. 3. Duplication of the right posterior cerebral artery (1PCA, 2PCA). The arrow signs the anastomosing vessel, which connected the 2PCA with the right internal carotid artery (ICA). PCoA - left posterior communicating artery, PCA - left posterior cerebral artery, BA basilar artery.

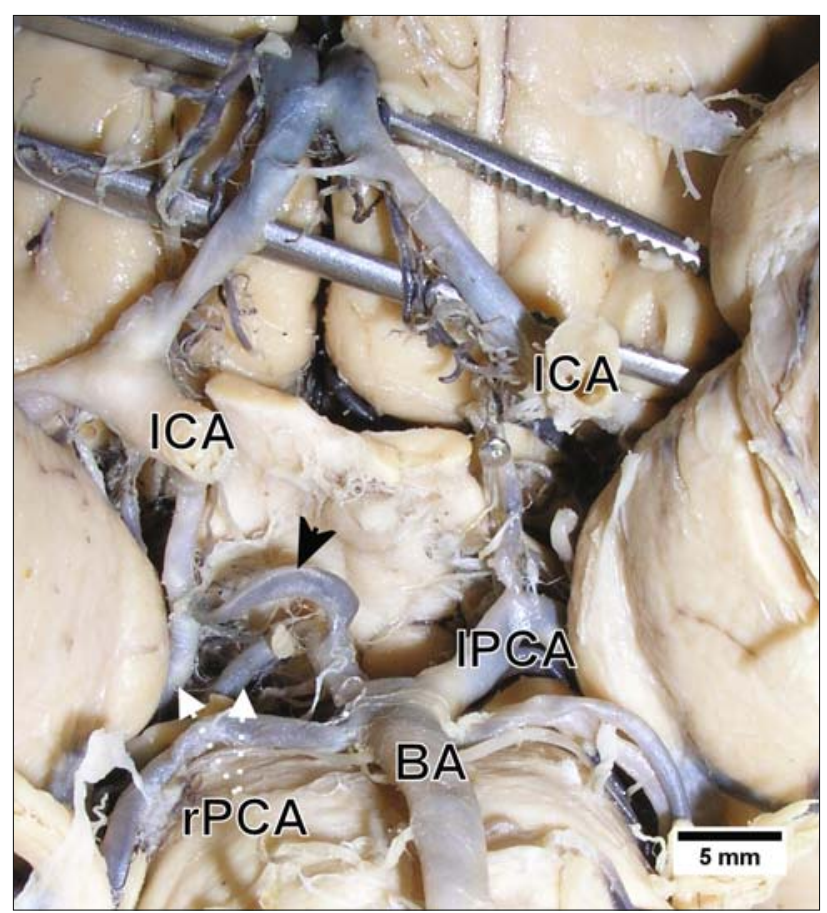

Fig. 4. Duplication of the right posterior cerebral artery (rPCA), the arrow head signs the communicating vessel, which interconnected the doubled arteries. ICA - internal carotid artery; IPCA - left posterior cerebral artery; BA - basilar artery. in three hemispheres for each side. The PcoA was fenestrated in one case at its attachment with the PCA (Fig. 2).

The infundibular dilatation at the attachment of PCoA with the internal carotid artery (ICA) was identified in 16 hemispheres with the average diameter of $2.5 \mathrm{~mm}$. It was detected bilaterally in four brains (2.16\%), unilaterally at the right side in five cases $(1.35 \%)$ and the left side in three cases (0.8\%).

The mean outer diameter of the right P1 was $1.78 \mathrm{~mm}$ with a maximal diameter of $2.9 \mathrm{~mm}$ and minimal diameter $0.67 \mathrm{~mm}$. The mean diameter of the left P1 was $1.87 \mathrm{~mm}$ with a range from 0.42 to $3.2 \mathrm{~mm}$. The right $\mathrm{P} 1$ segment was lesser than $0.8 \mathrm{~mm}$ in four cases (1\%), and the left P1 was hypoplastic in two cases (0.54 \%). The posterior cerebral artery (PCA) was doubled in two cases. In the first case: the first PCA originated from the ICA and had a higher caliber than that of the second PCA, which was branched from the basilar artery; the second PCoA was connected with ICA by an anastomosing vessel (Fig. 3). In the second case: the first and the second PCA originated from the ICA and BA respectively; a communicating vascular bridge joined the two arteries (Fig. 4).

The right-sided fetal pattern was found in 18 hemispheres (4.86\%), and the left-sided fetal pattern occurred in 13 hemispheres $(3.51 \%)$. The bilateral fetal pattern was noted in nine brains (4.86\%). The right-sided transitional pattern was observed in 11 cases (2.97\%), and the left-sided transitional pattern was seen in 13 cases (3.5\%). The transitional pattern occurred bilaterally in two brains (1\%). The adult pattern appeared on the right side in 42 hemispheres (11.35\%) and the left side in 38 hemispheres $(10.27 \%)$. The bilateral adult pattern occurred in 40 brains (21.62 \%). The results are summarized in Table 1 and Figure 5.

Tab. 1. The mean length and diameter \pm the standard error of the mean of the posterior communicating artery (PCoA), and precommunicating segment (P1) and postcommunicating segment (P2) of the posterior cerebral artery.

\begin{tabular}{lcc}
\hline & Mean length \pm SEM $(\mathrm{mm})$ & Mean diameter \pm SEM $(\mathrm{mm})$ \\
\hline Right PCoA & $10.62 \pm 0.24$ & $1.1 \pm 0.039$ \\
Left PCoA & $10.48 \pm 0.24$ & $1.04 \pm 0.035$ \\
Right P1 & $5.48 \pm 0.13$ & $1.78 \pm 0.038$ \\
Left P1 & $5.78 \pm 0.15$ & $1.87 \pm 0.038$ \\
Right P2 & - & $2 \pm 0.028$ \\
Left P2 & - & $2 \pm 0.03$ \\
\hline
\end{tabular}

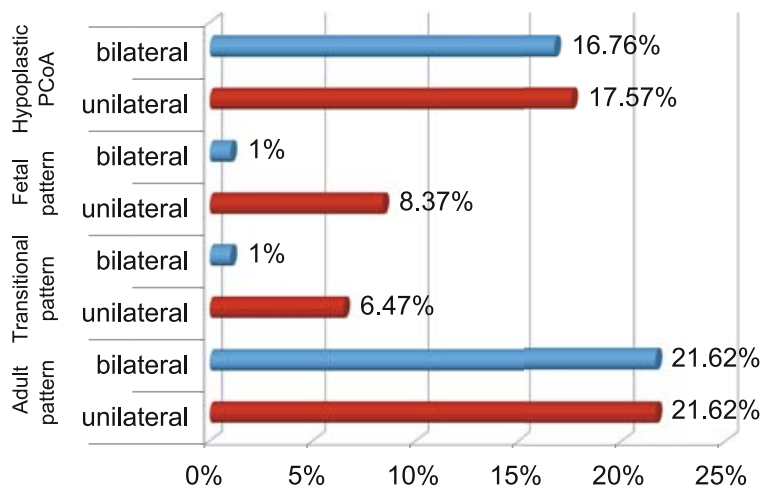

Fig. 5. The prevalence of the vascular patterns of the posterior part of the circulus arteriosus cerebri in $\mathbf{3 7 0}$ hemispheres 
Tab. 2. Comparison of some morphological studies of the posterior part of the circulus arteriosus cerebri.

\begin{tabular}{|c|c|c|c|c|c|}
\hline & Number of hemispheres & $\begin{array}{l}\text { Adult pattern } \\
\text { (\%) }\end{array}$ & $\begin{array}{c}\text { Transitional pattern } \\
\text { (\%) }\end{array}$ & $\begin{array}{c}\text { Fetal pattern } \\
(\%)\end{array}$ & $\begin{array}{c}\text { Hypoplastic PCoA } \\
\text { (\%) }\end{array}$ \\
\hline Saeki and Rhoton (1977) & 100 & 54 & - & 22 & 32 \\
\hline Al-Hussain (2001) & 100 & 77 & 8 & 15 & - \\
\hline Avci et al (2004) & 34 & 30 & 14 & 28 & 28 \\
\hline De Silva et al (2009) & 450 & 93.3 & 2.2 & 4.4 & - \\
\hline Present study (2018) & 370 & 43.24 & 7.47 & 13.22 & 34.58 \\
\hline
\end{tabular}

\section{Discussion}

The vertebral arteries emerge as a secondary development from two series of six cervical intersegmental arteries. These vessels provide the proximal supply to the longitudinal neural arteries. The two longitudinal neural arteries consolidate across the midline to form the basilar artery (BA). The ICA develops from the third aortic arch and the cranial portion of the dorsal aorta. The anastomotic connections between the vertebrobasilar and internal carotid arterial systems create the CW $(11,12,13)$. Anomalies and changes of the developmental process during the embryological period may cause numerous variations of caliber and configuration of the brain arteries. These anatomical variations may be attributed to: 1 . the choice of unusual paths in the primitive vascular plexus; 2. the persistence of vessels usually obliterated; 3 . the disappearance of vessels usually retained; 4 . incomplete development; and 5 . fusion and absorption of parts usually different $(11,14)$.

The variations of the CW are the consequences of developmental modifications. The statistical analysis established the relationship between the stage of the brain development and the forming of the diverse configurations. The transitional pattern, characterized by the equal diameter of P1 and PCoA appears early in the development. The frequency of the appearance of the fetal and adult patterns increases during development at the expanse of the transitional pattern due to the rapid growth of the occipital lobe (15).

The caudal branch of the primitive ICA creates a plexiform structure preceding its incorporation into the PCoA and PCA. This plexus may persist postnatally as the single intermediate communicating artery, which explains the origin of the vascular anastomosis joining the doubled PCA. The persistence of the vestigial vessels of the internal carotid and vertebrobasilar systems or the carotid-basilar anastomoses explains the cases of vascular duplication and fenestration $(14,16)$.

The prevalence of the vascular configurations of the posterior part of the CW reported in the literature showed a vast range. The incidence of the fetal pattern varied from 15 to $40 \%$ of cases. The transitional pattern appeared in $8-18 \%$ of cases, and the adult pattern was observed in 30-77\% of cases. Hypoplasia of the PCoA was varied from 18 to $32 \%$ of cases. The right PCoA was absent in 2.2-4.5\%, and the left artery was absent in 0.85-6.5 \% of cases $(5,6,15,17,18,19)$. The present study showed that the prevalence of the fetal pattern, including the bilateral and unilateral occurrence was lower than the range of the previous studies (13.23 \%). The unilateral and the bilateral transitional pattern were identified in about $7.47 \%$ of the cases. The adult pattern was the most commonly observed vascular configuration in the present study (43.24\%). The occurrence of the PCoA hypoplasia was higher than the range specified in literature (34.33\%). The rate of the PCoA aplasia was lower than the range described by the previous studies ( $0.81 \%$ for each side). Table 2 is presenting a compression of some morphological studies of the posterior part of the CW.

The PCoA infundibulum is a funnel-shaped dilatation of the attachment of the PCoA with ICA. This dilatation can be confused with an aneurysm during the surgical procedures. It was pointed out in the previous studies in the range from 6 to $22 \%(6,20,21$, $22)$. The incidence of the infundibular dilatation in the present work was $4.31 \%$ of cases.

The vascular diameter is not constant, and it can change to a minor extent during the time. Therefore, the outer diameter of the hypoplastic artery can rise over time. The post-mortem measurement is carried out on empty and compressed vessels, which can cause differences in the measured vascular calibers among the radiological and morphological studies $(9,23)$.

As the calibers of the cerebral vessels differ amongst the general population and as the resistance to the blood flow of a vessel is proportional to the fourth power of the vessel diameter, a minor change in the vascular diameter may lead to a profound influence on the ability to provide collateral flow. The vascular threshold diameter permitting the collateral flow through the CW lies between $0.4-0.6 \mathrm{~mm}(8,24)$.

The morphological anomalies of the CW were considered a risk factor of the cerebral aneurysms, hemorrhage, and ischemia. The disorder of the anastomosis between the carotid and vertebrobasilar systems may cause disturbances in the blood flow to individual parts of the brain (25). The different patterns of the posterior part of the CW may cause several consequences from the clinical point of view, which receives its blood supply from the posterior cerebral artery such as: thalamus, mesencephalon and occipital lobe. The hypoplastic PCoA becomes a risk factor for the ischemic cerebral stroke in the case of presence of ipsilateral occlusion of the ICA $(5,26,27)$. The incidence of aneurysm development in the fetal and transitional pattern, and in the case of hypoplastic PCoA, is higher than in the adult pattern (5). Owing to the adjacent topographical relation of the PCoA and the oculomotor nerve, the PCoA an aneurysm is responsible for 13-30\% of the acquired third cranial nerve palsies (28).

The circulus arteriosus cerebri Willisi presents a unique vascular system, which permits a degree of collateral blood flow. Numerous factors including genetic and environmental factors may affect the development of vessels and their dimensions. Therefore, 
variable vascular patterns are formed with physiological or even pathological effect on the hemodynamics and collateral function of the cerebral vessels. The detecting of the vascular dimensions in vivo can help in the prediction and may be the prevention of possible cerebrovascular pathologies in the future. Correlation and interdisciplinary cooperation of the studies dealing with morphology, radiology, and hemodynamics of the cerebral vessels are becoming an urgent need. The assumed results of this cooperation can be used in tabulating the calibers of the cerebral vessels and determining the threshold dimensions, under which failure of hemodynamics and collateral function may appear.

\section{References}

1. Standring S (Ed). Gray's Anatomy: The Anatomical basis of Clinical Practice. $40^{\text {th }}$ edition. New York: Elsevier, Churchill Livingstone, 2008: $1-1551$.

2. Saha A, Bhagyalakshmi B, Mandal S, Banopadhyaya M. Variation of posterior communicating artery in human brain: a morphological study. Gomal J Med Sci 2013; 11 (1): 42-46.

3. van Raamt AF, Mali WP, van Laar PJ, van der Graaf Y. The Fetal Variant of the Circle of Willis and its Influence on the cerebral collateral circulation. Cerebrovasc Dis 2006; 22 (4): 217-224.

4. Burlakoti A, Kumaratilake J, Taylor J, Massy-Westropp N, Henneberg M. The cerebral basal arterial network: morphometry of inflow and outflow components. J Anat 2017; 230 (6): 833-841.

5. De Silva KRD, Silva TRN, Gunasekera WSL, Jayesekera RW. Variation in the origin of the posterior cerebral artery in adult Sri Lankans. Neurol India 2009; 57 (1): 46-49.

6. Avci E, Baskaya MK. The surgical anatomy of the anomalous posterior communicating artery. International Congress Series 2004; 1259: 3-10.

7. Bender M, Olivi A, Tamargo RJ. Iulius Casserius and the first anatomically correct depiction of the circulus arteriosus cerebri (of Willis). World Neurosurger 2013; 79 (5-6): 791-797.

8. Hoksbergen AWJ, Fülesdi B, Legemate DA, Csiba L. The collateral configuration of the circle of Willis: transcranial color-coded duplex ultrasonography and comparison with postmortem anatomy. Stroke 2000; 31 (6): 1346-1351.

9. Eftekhar B, Dadmehr M, Ansari S, Ghodsi M, Nazparvar B, Ketabchi E. Are the distributions of variations of circle of Willis different in different populations? - Results of an anatomical study and review of literature. BMC Neurology 2006; 6 (22). www.biomedcentral.com/1471$2377 / 6 / 22$

10. Loukas M, Louis Jr RG, Childs RS. Anatomical examination of the recurrent artery of Heubner. Clin Anat 2006; 19 (1): 25-31.

11. Arey LB. Developmental anatomy, 7th edition. Philadelphia: Saunders, 1965: 1-695.
12. Okahara M, Kiyosue H, Mori H, Tanoue S, Sainou M, Nagatomi H. Anatomic variations of the cerebral arteries and their embryology: a pictorial review. Europ Radiol 2002, 12: 2548-2561.

13. Sadler TW. Longman's Medical Embryology. Baltimore: Williams and Wilkins, 1990: 1-411.

14. Vasovic L, Milenkovic Z, Pavlovic S. Comparative morphological variations and abnormalities of circles of Willis: a minireview including two personal cases. Neurosurg Rev 2002; 25 (4): 247-251.

15. Van Overbeeke JJ, Hillen B, Tullekena CAF. Comparative study of the circle of Willis in fetal and adult life. The configuration of the posterior bifurcation of the posterior communicating artery. J Anat 1991; 176: 45-54.

16. Vasović L, Trandafilović M, Jovanović I et al. An excess vessel in the posterior part of the human cerebral arterial circle (CAC): a case series. BMC Neurology 2010; 10 (53). www.biomedcentral.com/14712377/10/53

17. Al-Hussain SM, Shoter AM, Bataina ZM. Circle of Willis in adults. Saudi Med J 2001; 22 (10): 895-898.

18. Saeki N, Rhoton AL Jr. Microsurgical anatomy of the upper basilar artery and the posterior circle of Willis. J Neurosurg 1977; 46 (5): 563-578.

19. Karatas A, Yilmaz H, Coban G, Koker M, Uz A. The Anatomy of Circulus Arteriosus Cerebri (Circle of Willis): A Study in Turkish Population. Turk Neurosurg 2016; 26 (1): 54-61.

20. Bisaria KK. Anomalies of the posterior communicating artery and their potential clinical significance. J Neurosurg 1984; 60 (3): 572-576.

21. Hassler O, Saltzman GF. Angiographic and histologic changes in infundibular widening of the posterior communicating artery. Acta Radiol Diagn 1963; 1: 321-327.

22. Baskaya MK, Coscarella E, Gomez F, Morcos JJ. Microsurgical and angiographic anatomy of the posterior communicating and anterior choroidal arteries. Neuroanat 2004; 3: 38-42.

23. Papantchev V, Hristov S, Todorova D et al. Some variations of the circle of Willis, important for cerebral protection in aortic surgery - a study in Eastern Europeans. Europ J Cardio-thorac Surg 2007; 31: 982-989.

24. David T, Moore S. Modeling perfusion in the cerebral vasculature. Med Engineer Phys 2008; 30: 1227-1245.

25. Degani S. Evaluation of Fetal Cerebrovascular Circulation and Brain Development: The Role of Ultrasound and Doppler. Semin Perinatol 2009; 33 (4): 259-269.

26. Krishnamurthy A, Nayak SR, Ganesh Kumar C. Morphometry of posterior cerebral artery: embryological and clinical significance. Roman J Morphol Embryol 2008; 49 (1): 43-45.

27. Chuang YM, Liu CY, Pan PJ, Lin CP. Posterior communicating artery hypoplasia as a risk factor for acute ischemic stroke in the absence of carotid artery occlusion. J Clin Neurosci 2008; 15 (12): 1376-1381.

28. Sahni D, Jit I, Lal V. Variations and anomalies of the posterior communicating artery in northwest Indian brains. Surg Neurol 2007; 68 (4): 449-453. 